\section{The protective effects of aqueous extract of Carica papaya seeds in paracetamol induced} nephrotoxicity in male wistar rats

Madinah Naggayi ${ }^{1}$, Nozmo Mukiibi ${ }^{1}$, Ezekiel Iliya ${ }^{2}$

1. Mbarara university of science and technology, Physiology

2. Ahmad Bello University, Physiology

\section{Abstract}

Background: Oxidative stress plays a crucial role in the development of drug induced nephrotoxicity. The study aimed to determine the nephroprotective and ameliorative effects of Carica papaya seed extract in paracetamol-induced nephrotoxicity in rats.

icity in rats.

Objectives: To carry out phytochemical screening of Carica papaya, measure serum urea, creatinine and uric acid and describe the histopathological status of the kidneys in the treated and untreated groups.

Methods: Phytochemical screening of the extract was done. Thirty two adult male Wistar rats were divided into fou groups ( $\mathrm{n}=8$ in each group). Group A (control) animals received normal saline for seven days, group B (paracetamol group) received normal saline, and paracetamol single dose on the 8th day. Group C received Carica papaya extract (CPE) $500 \mathrm{mg} /$ $\mathrm{kg}$, and paracetamol on the 8th day, while group D, rats were pretreated with CPE $750 \mathrm{mg} / \mathrm{kg} /$ day,and paracetamol administration on the 8th day. Samples of kidney tissue were removed for histopathological examination.

Results: Screening of Carica papaya showed presence of nephroprotective pytochemicals. Paracetamol administration resulted in significant elevation of renal function markers. CPE ameliorated the effect of paracetamol by reducing the markers as well as reversing the paracetamol-induced changes in kidney architecture.

Conclusion: Carica papaya contains nephroprotective phytochemicals and may be useful in preventing kidney damage induced by paracetamol.

Keywords: paracetamol,papaya, kidney.

\section{DOI: http://dx.doi.org/10.4314/ahs.v15i2.37}

\section{Introduction}

Paracetamol (acetaminophen) is one of the most popular and widely used drugs for the treatment of pain and fever. It occupies a unique position among analgesic drugs. Unlike NSAIDs it is almost unanimously considered to have no anti-inflammatory activity and does not produce gastrointestinal damage ${ }^{1}$. Paracetamol is contained in many preparations, available as both over-the-counter and as prescription-only medications. Because of its wide availability paired with comparably high toxicity, (compared to ibuprofen and aspirin) there is a much higher potential for overdose ${ }^{2}$. Paracetamol is especially dangerous on the liver if taken over long pe-

\section{Corresponding author:}

Madinah Naggayi,

Mbarara university of science

and technology, Physiology

Tel: +256774 422964 ,

+256704 422964

Email: naggmad@gmail.com the natural antioxidant defenses of the body are overwhelmed, and the liver is damaged by the buildup of dangerous free radicals. Although nephrotoxicity is less common than hepatotoxicity in paracetamol overdose, renal tubular damage and acute renal failure can occu even in the absence of liver injury ${ }^{4}$, and can even lead to death in humans and experimental animals, ${ }^{5}$. Even at normal NSAID dosages, people with compromised kidney function can develop NSAID toxicity ${ }^{6}$.

Studies are going on throughout the world for the search of protective molecules that would provide maximum protection to the liver, kidney as well as other organs and practically very little or no side effects would be exerted during their function in the body $y^{7,8,9}$. A number of herbs are traditionally used in different countries for mitigation of drug or toxin induced hepatic and renal disorders $^{10}$

Papaya tree is grown extensively all over the tropical regions including Uganda and it is cultivated for its fruits and latex papain, an enzyme that is used in the food riods $^{3}$. If toxic levels of paracetamol occur in the liver, industry. The papaya, papaw, or pawpaw is the fruit of the plant Carica papaya, the common species in the genus Carica of the plant family Caricaceae. Papaya is a fruit widely consumed and served either green or ripe. Its juice is also a popular beverage, while its leaves and young stems are sometimes steamed and served as a vegetable. Papava has digestive, antibacterial, anti-inflammatory, anti-ageing, anti proliferative, contraceptive capability and its anti-oxidant nature helps to reduce the likelihood of atherosclerosis, strokes, diabetes, and heart attacks ${ }^{11}$

The prophylactic action of papaya was observed in rats with acetaminophen induced hepatotoxicity ${ }^{12}$. However, the effect of papaya on acetaminophen induced nephrotoxicity had not yet been studied. The present study was therefore designed to investigate protective effects of papaya against acetaminophen induced nephrotoxicity.

The effect of the plant extract in paracetamol induced toxicity was evaluated by determining the levels of creatinine, urea and uric acid from the serum in all the experimental groups. To assess the ultra structural change of the kidney, histopathological studies were conducted from kidney sections of normal rat, rat treated with paracetamol, and rat treated with plant extract prior and later with paracetamol.

\section{Materials and methods}

\section{Collection of Plant materials}

Fifty mature, unripe fruits of Carica papaya were collected around Mbarara, Western Uganda, in the month of May, 2012. These were taken to the Biology Department in Faculty of science, Mbarara University where the plant was identified and authenticated by Dr. Eunice Olet. A reference specimen (Naggayi 001/2012) of this plant was retained in the department.

\section{Preparation of plant materials}

The pawpaw fruits were cut into pieces and the wet seeds separated out. These were then gently washed and rinsed in tap water thoroughly and completely air-dried at room temperature for 4 weeks. The dried seeds were pulverized into a coarse powder using a mechanical grinder. The powder was passed through a sieve $150 \mu \mathrm{m}$ so as to obtain a very fine powder. $344 \mathrm{~g}$ of the pow dered dried seeds of Carica papaya was cold macerat- in $1000 \mathrm{ml}$ of distilled water in a water bath for 72 hours with frequent stirring, after which it was filtered through a piece of clean cotton gauze. The residue was re-washed three times with this same quantity of solvent and filtered. The filtrate was further re-filtered using Whatman filter paper no.1 and the final filtrate was completely oven-dried at a preset temperature of 60 ${ }^{\circ} \mathrm{C}$ for a week, producing a fine aromatic and chocolate color solid residue (the dry aqueous seed extract). The dry residue obtained was weighed and stored in an air and water-proof container kept in a refrigerator at $4^{\circ} \mathrm{C}$.

\section{Animals}

32 adult male Wistar rats, weighing between 150 and $180 \mathrm{~g}$ body weight were used for this study. The rats housed in wooden animal cages $(38 \times 23 \times 10 \mathrm{~cm})$ in groups of 8 animals with $12: 12 \mathrm{hr}$ of light: dark cycle and maintained under standard laboratory conditions (temperature $25 \pm 2^{\circ} \mathrm{C}$ ). The animals were fed with standard rodent diet "mice pencils" from Nuvita animal feed. They were maintained under normal laboratory temperature, and provided water ad libitum. They were used for the study after one week of acclimatization. They were fasted 14 hours (6: 00 p.m- 8: 00 a.m) before the experiments, but were allowed free access to water. Approval of institutional review ethical committee was obtained prior to the experiments and the experimental rats were all handled in strict compliance with international guidelines as prescribed by the Canadian council on the care and use of laboratory animals in biomedical research (1984)

Chemicals used: All reagents and drugs used were obtained commercially and were of analytical grade. Paracetamol was purchased from Kampala Pharmaceutical Industries manufactured by Hebei Jiheng (Group Pharmaceutical Co., Ltd.), dissolved in normal saline and administered at a concentration of $100 \mathrm{mg} / \mathrm{ml}$

Phytochemical procedure: Phytochemical screening: A preliminary phytochemical screening of the seed extract of Carica papaya was also done using standard methods of analysis (13).

Dose administration and induction of nephrotoxicity

Animals were randomly assigned into four groups (A-D) of eight rats per group (14). Group (A) animals served as the control group and were treated with normal sa- 
line $(5 \mathrm{ml} / \mathrm{kg}$ by gavage daily) throughout the experi- identify the effect of the treatment on the biochemical were also trented with nom andine $(5 \mathrm{~m} / / \mathrm{k}$ age) daily for 7 days. Animals in group (C) were treated with $500 \mathrm{mg} / \mathrm{kg}$ body weight of the aqueous extract of Carica papaya seed once daily by gavage for 7 days and group (D) animals were treated with $750 \mathrm{mg} / \mathrm{kg}$ body weight of the aqueous extract of Carica papaya once daily by gavage for 7 days. On the 8 th day, paracetamol $1 \mathrm{~g} / \mathrm{kg}$ body weight $\mathrm{t}^{15}$ to all the animals except the animals in group (A). Treatments were done between 9.00 and $10.00 \mathrm{a} . \mathrm{m}$ to minimize possible diurnal effects.

\section{Sampling and biochemical analysis}

Forty-eight hours after paracetamol administration 7 , rats were subjected to diethyl ether anesthesia after which blood samples were collected in vacutainer tubes for evaluating the serum biochemical parameters. Blood samples were collected by cardiac puncture with $21 \mathrm{G}$ needle mounted on $5 \mathrm{ml}$ disposable syringe. $4 \mathrm{mls}$ of blood were collected from each animal. Blood was left to clot to separate the clotting factors from the sera for an hour at room temperature and then centrifuged for $10 \mathrm{~min}$ at $3000 \mathrm{rpm}$. The obtained clear sera was stored at $-20^{\circ} \mathrm{C}$ for subsequent measurement of blood urea, creatinine and uric acid levels using commercially available kits prepared by Human Company and an automated analyzer (Humastar 180), according to the manufacturer's instructions.

\section{Histopathological examination}

Samples of kidney tissue from each group were fixed immediately in 10\% formalin for a period of 48 hours, the pathological symptoms of nephrotoxicity such as leukocyte infiltration.

\section{Data analysis}

Data analysis was done using Graph Pad Prism 5. Results were expressed as mean \pm standard error of the mean (SEM) and these were used to determine the differences in the serum urea, creatinine and uric acid quantities in the different groups. A One-Way ANOVA was done followed by Newman-Keuls Multiple Comparison test set at a significant level of $\mathrm{p} \leq 0.05$ to com-
pare the mean values of the different groups in order to pectively. Graphs for the representation of the results were also drawn using graph pad software.

Results

Phytochemical screening

The crude extract of Carica papaya seeds was tested for the most common phytochemical constituents of medicinal plants for which nephroprotective activity of other plants have been attributed. These included; saponins, tannins, flavonols, glycosides, terpenoids, alkaloids, reducing sugars, steroids, proteins, fats and polyphenols. The results showed that the most abundant phytochemicals were tannins, alkaloids, phenols, vitamins, flavonoids and terpenoids as shown in table 1

Table 1: Phytochemical constituents of C. papaya eed aqueous extract

\begin{tabular}{|lc|}
\hline Phytochemical constituent & Level \\
Saponins & + \\
Tannins & +++ \\
Flavonols & ++ \\
Glycosides & + \\
Terpenoids & ++ \\
Alkaloids & +++ \\
Reducing sugars & + \\
Amino acids & ++ \\
Fats & + \\
Proteins & ++ \\
Phenols & +++ \\
Vitamins & +++ \\
Sterols and Triterpenes & ++ \\
\hline
\end{tabular}

Key: +++ abundant, ++ moderate, + trace

\section{Biochemical markers.}

Serum creatinine, uric acid and urea concentrations were significantly increased $(p<0.0001)$ in paracetamol treated group of animals compared to the normal animals indicating the induction of severe nephrotoxicity. (Fig.1, 2 \& 3). Treatment with the aqueous extract of Carica papaya showed significant decrease in concentrations of serum creatinine, uric acid and urea compared suspension was given by oral route at a single dose of dehydrated in graded (50-100\%) alcohol, embedded in paraffin, cut into $5 \mu \mathrm{m}$ thick sections and stained with Hematoxylin-eosin. These sections were evaluated for

Comparison between the control group (group A) and atinine concentrations (Fig. 1), neither did comparison the groups that were treated with the extracts (group $\mathrm{C}$ between the control group (A) and group (D) show any and D) did not show any significant difference in cieto the paracetamol treated group. 
Figure 3 shows that comparison between the extract at difference although the urea levels were slightly lower $500 \mathrm{mg} / \mathrm{kg}$ and $750 \mathrm{mg} / \mathrm{kg}$ also showed a significant dif- in the $500 \mathrm{mg} / \mathrm{kg}$ extract than for the control group. It ference as well as between the control (group A) and is therefore concluded from current results that Carica group $\mathrm{D}$ while comparison between the control group papaya is useful in protecting against paracetamol inand the extract at $500 \mathrm{mg} / \mathrm{kg}$ did not show a significant duced nephrotoxicity.

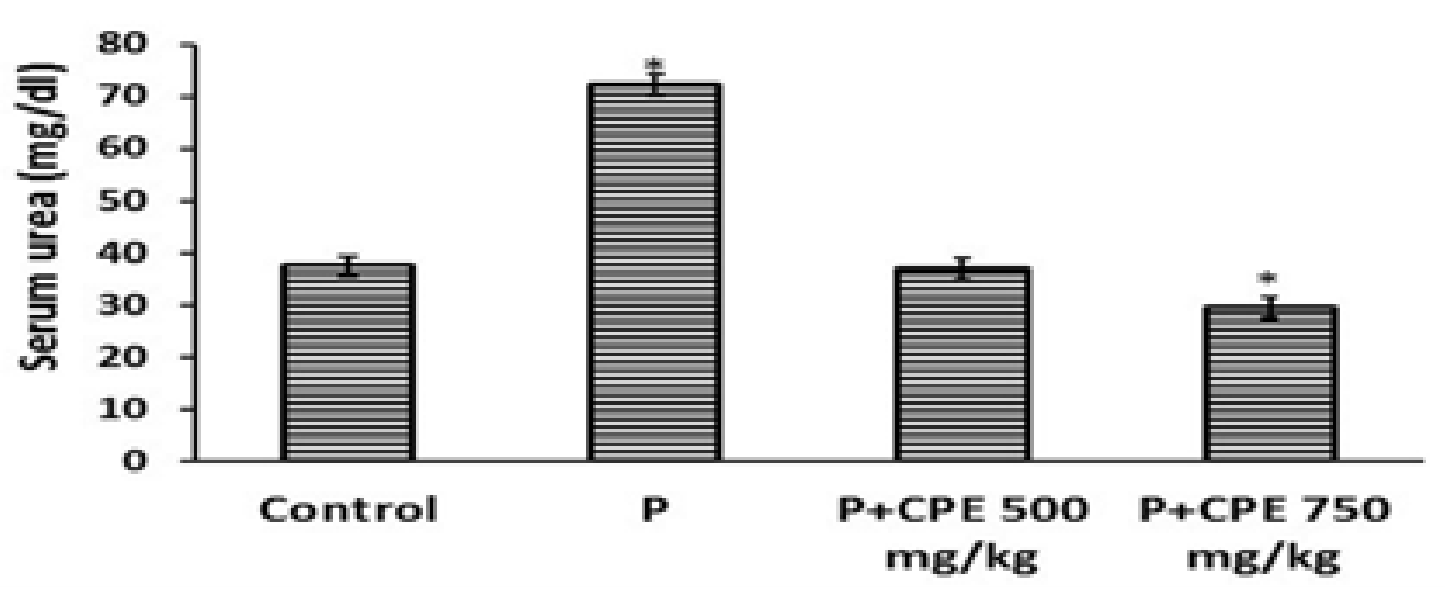

Fig.3. Effect of pre-treatment with aqueous seed extract of Carica papaya on urea levels
in rats with Paracetamol-induced nephrotoxicity Control (Saline) $P$ (Paracetamol: $1 \mathrm{gm} / \mathrm{kg}$ ) CPE (Carica papaya extract). All values are presented as mean + SEM. $(\mathrm{n}=8)$. The asterisk shows statistical significance with respect to control $(\mathrm{p} \leq 0.05)$.

\section{Histopathological evaluation}

flat (squamous) epithelium (Arrow). Note the uniform Biochemical results were confirmed by histological space between glomerulus and capsule wall. (Bowman's patterns of normal kidney in Figure 4A which shows Space BS). The proximal renal tubules (PT) are lined intact lobular organization of the glomeruli (GL) and with typical cuboidal epithelium with a brush border surrounding Bowman's capsule (BC) which is lined by a The distal tubules (DT) have a relatively regular distinct

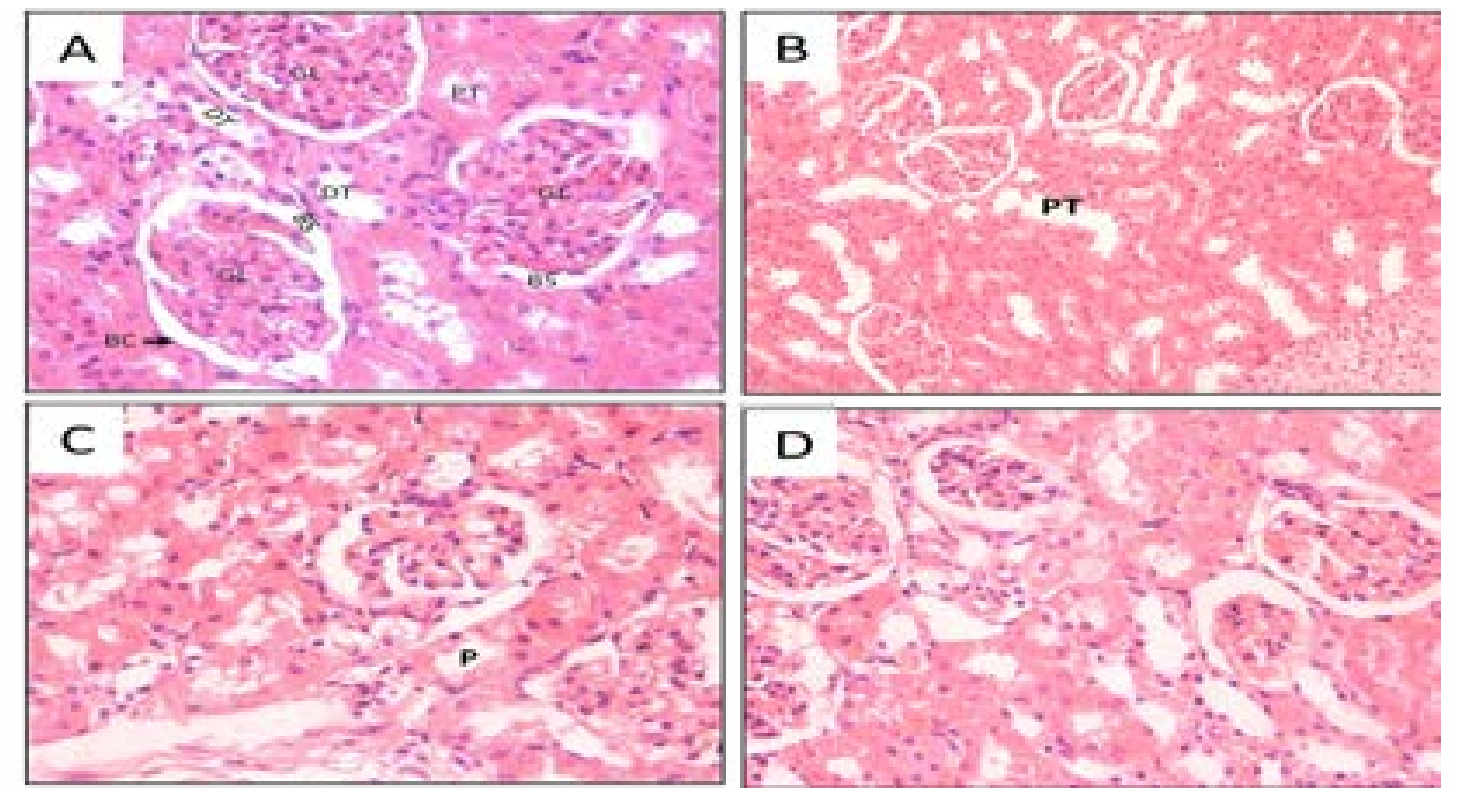

Figure 4. Kidney sections stained with Hematoxylin-Eosin (Magnification $x$ 40o). A)
negative control, B) positive control (paracetamol), C) CPE 500 mg/kg, D) CPE negative control, B) positive control (Paracetamol), C) CPE $500 \mathrm{mg} / \mathrm{kg}$, D) CPE
$750 \mathrm{~g} / \mathrm{kg}$. GL G Glomerulus; BC - Bowman's Capsule; BS - Bowman's Space; DT - Distal
convoluted tubule; PT - Proximal convoluted tubule.

Figure $4 \mathrm{~B}$ shows enlarged glomeruli and some loss of surrounding Bowman's capsule. Paracetamol also caused hypercellularity in Bowman's capsule indicating leukocyte infiltration, shrunken glomerulus or glomerular atrophy. Some cells of the tubular epithelium show features of severe tubular necrosis. Interstitial edema was also exhibited due to leakage of fluid from the tubules as a result of the toxic effects of paracetamol. Proximal tubules were dilated with loss of cellular boundary and loss of brush border $(\mathrm{P})$, thus debris and granules from the epithelial cells leaked into the tubula lumen.

Figure 4C shows Bowman's capsule with moderate leukocyte infiltration. Asymmetry was still noted in some of the glomeruli. There are also diffuse mildly swollen tubules. Moderate necrosis and degeneration are shown. Proximal tubules $(\mathrm{P})$ show dilatation, and there is brush border in some of them. Since approximately $70 \%$ of the glomerular filtrate is normally reabsorbed from the proximal tubules, this function being reflected in the structure of the epithelial lining, the appearance of brush border in some tubules implies improvement in the functioning of the nephrons. This was reflected in the reduction of the biochemical markers in blood of Group C animals hence the ameliorative effect of papaya although some debris was still observed in some tubules bringing about the dilatation.

Figure 4D shows no obvious tubular cast deposition, and brush border were observed in the majority of proximal tubules (PT) and intraluminal cell debris were absent.

\section{Discussion}

Various environmental toxicants and clinically useful drugs, like paracetamol and gentamicin, can cause severe organ toxicities through the metabolic activation to highly reactive free radicals including the superoxides and oxygen reactive species ${ }^{16}$. The selective renal accumulation of non-steroidal anti-inflammatory nephrotoxins including paracetamol in animal and human is thought to result in a chain of biochemical reactions which culminate in acute or chronic nephropathies In addition, paracetamol has been reported to promote hepatocyte and renal apoptosis ${ }^{18,19}$. Paracetamol toxic overdose is often manifested by too many metabolic disorders including serum electrolytes, urea, creatinine nd uric acid derangements. Serum urea and creatinine considered the major nephrotoxicity markers ${ }^{20}$ although serum urea concentration is often considered a more reliable renal function predictor than serum creatinine ${ }^{7}$. Blood urea nitrogen is found in the liver protein that is derived from diet or tissue sources and is normally excreted in the urine. Creatinine, on the other hand, is mostly derived from endogenous sources by tissue creatine breakdown ${ }^{21}$. In the present study, administration of a nephrotoxic dose of paracetamol to rats resulted in a significant elevation of serum levels of urea, creatinine and uric acid in paracetamol administrated group within 48 hours of exposure to it when compared to the normal control group.

These results are in agreement with those observed in Isik $\mathrm{B}$ et. $\mathrm{a}^{15}$ who noticed an elevation in serum urea and creatinine in rats after $1 \mathrm{~g} / \mathrm{kg}$ body weight of paracetamol administration. Moreover an elevation in serum urea and creatinine in a woman following therapeutic dose of paracetamol three days before hospital admission was reported ${ }^{22}$. This elevation in the levels of urea and creatinine was explained by the presence of strong correlation between nephrotoxicity and oxidative stress ${ }^{23,14}$. These biochemical alterations were corroborated by the histological findings of glomerular and tubulo-interstitial necrosis in the untreated model control group (B). However, daily pretreatment with $\mathrm{CPE}$ for 7 days conferred nephroprotection in the paracetamol renal injured rats in a dose-dependent fashion and $750 \mathrm{mg} / \mathrm{kg}$ dose offered maximum protection.

The nephroprotective property of the extract was therefore confirmed by significant improvement of the kidney architecture by reversing the nephrotoxic effects of paracetamol such as glomerular congestion, interstitium with inflammatory cells, tubular necrosis, peritubular necrosis and presence of intra-luminal casts suggesting massive total necrosis. Although, the possible mechanism(s) of its protection against paracetamol induced nephrotoxicity was not studied in the current study, it is possible that the protective effect of the extract is mediated through antioxidant and/or free radical scavenging activities. Literature has shown medicinal plants with nephroprotective properties to mediate their protection via antioxidant and/or free radical scavenging activities due to the high concentration of flavonoids and alkaloids they contain ${ }^{24,25}$. This is in agreement with the findings of this study. Summing these facts, it is plausi- 
le for the alkaloid, flavonoid and saponin components of CPE to be responsible for the observed biological effects. Flavonoids, tannins, and saponins have been reported to exert profound in vitro and in vivo stabilizing effect on the lysosomes of experimental animals ${ }^{26}$. Plant flavonoids which show an antioxidant activity in vitro also function as antioxidants in vivo. Tannins and saponins stabilize the erythrocyte membrane by binding cations and other biomolecules ${ }^{27}$. Phenolic compounds function as high-level antioxidants because they posses the ability to absorb and neutralize free radicals as well as quench reactive oxygen species. Again, a strong relationship between the total phenolic content and antiox-

idant activity in fruits, vegetables, grain products, and plant subjects of ethno-pharmacological treatments has also been reported ${ }^{28}$

\section{Conclusion}

The aqueous extract of Carica papaya seeds produced adequate nephroprotective activity on albino Wistar rats as evidenced by the reduction in the biochemical parameters and improvement of the kidney architecture. This supported the folklore use of the title plant in renal disorders.

It is likely that the nephroprotective activities of the aqueous seed extract of the unripe mature fruits of Carica papaya in paracetamol-induced nephrotoxicity may involve its antioxidant and/or oxidative free radical scavenging activities which are provided for by the combined effect of active principles present in it.

However:

1. Financial limitations restricted us from investigating oxidative stress markers as one of the common pathogenic mechanisms for drug induced nephrotoxicity, thus future studies about the protective effects of papaya via antioxidants in ameliorating kidney damage are recommended.

2. The present study does not show the type of active phytochemicals responsible for the nephroprotective property of Carica papaya seed extract and this may be considered as one of the scope for future study on the plant.

\section{Acknowledgements}

I would like to express my very great appreciation to Mr. Joseph Oloro for his valuable and constructive suggestions during the planning and development of this research work. My thanks also go to the technicians of he MUST clinical/research laboratory, histology laboratory of the anatomy department as well as th macy Laboratory for allowing me to use the equipment in their facilities.

This work was made possible by medical education for equitable services to all Ugandans, a medical education partnership initiative grant number 5R24TW008886 from the office of olobal AIDS coordinator and the

U. S. department of health and human services, health resources and services administration and national institutes of health. Its contents are solely the responsibility of the authors and do not necessarily represent the official views of the government.

\section{References}

1. Bertolini A, Ferrari A, Ottani A, Guerzoni S, Tacchi $\mathrm{R}$, Leone S. Paracetamol: new vistas of an old drug. Fall-Winter. 2006;12 (3-4):250-75,

2. Sheen C, Dillon J, Bateman D, Simpson K, Macdonald T. "Paracetamol toxicity: epidemiology, prevention and costs to the health-care system". QJM: Monthly journal of the Association of Physicians. 2002; 95 (9):609-19.

3. Ford MD, and Mc Martin K. Ethylene glycol and methanol. In: Ford M.D, Delaney K.A., Ling L.J., et. al., editors. Clinical toxicology. 1st edition. Philadelphia 7 WB Saunders. 2001; pp 757-67.

4. Jones AF, Vale JA. Paracetamol poisoning and the kidney. Journal of Clinical Pharmacy and Therapeutics, 1993;18 (1): 5-8.

5. Sarumathy K. A protective effect of Caesalpinia sappan on acetaminophen induced nephrotoxicity and oxidative stress in male albino rats. IJPI's journal of pharmacology and toxicology. 2011; $1: 2$

6. Whelton A, Hamilton CW. Non steroidal anti-inflammatory drugs: Effects on kidney function. Journal of Clinical Pharmacology. 1991; 31 (7):588-98.

7. Palani SS, Raja R, Kumar P, Jayakumar S. Therapeutic efficacy of Pimpinella tirupatiensis (Apiaceae) on acetaminophen induced nephrotoxicity and oxidative stress in male albino rats. International Journal Pharm Tech. 2009. 8. Montilla P., Barcos M., Munoz M.C, Bujalance, Munoz-Castaneda J.R., and Tunez I. Red wine prevents brain oxidative stress and nephropathy in streptozotocin-induced diabetic rats. J. Biochem. Mol. Biol. 2005; 38 539-544.

9. Mansour HH, Hafez HF, Fahmy NM. Silymarin modulates Cisplatin-induced oxidative stress and hepatotoxicity in rats. J.Biochem. Mol. Biol. 2006; 39, 656-661.
10. El-Beshbishy HA. Hepatoprotective effect of green tea [Camellia sinensis] extract against tamoxifen-induced liver injury in rats. J. Biochem. Mol. Biol. 2005; 38 300-306.

11. Aravind G1, Debjit B1, Duraivel S1, Harish.G1.Traditional and medicinal uses of Carica papaya. Journal of medicinal plants studies.2013; 1: 7-15.

12. Srinivas K. Influence of Carica papaya Linn Extracts on Paracetamol and thioacetamide Induced Hepatic Damage in Rats. The internet journal of pharmacology. 2009; 9: number 1.

13. Trease GE, Evans WC. Pharmacognosy. 15th Ed. London: Saunders Publishers. 2002; pp. 42-44. 221229, 246-249, 304-306, 331-332, 391-393.

14. Ajami M, Shahriar E, Hamidreza PTC, Rouhollah $\mathrm{H}$, Soltan AE. Effect of Crocus sativus on gentamicin induced nephrotoxicity. Biological Research. 2010; 43 83-90

15. Isik B, Bayrak R, Akcay A. Erdosteine against acetaminophen induced renal toxicity. Molecular Cell Biochemistry.2006; 287: 185-91

16. Abraham P and Wilfred G. Oxidative damage to the lipids and proteins of the lungs, testis and kidneys of rats during carbon tetrachloride intoxication. Clinical Chim Acta. 1999; 289: 177 - 179.

17. Schnellman RG. Toxic responses of the kidney. In: Casarett and Doull's Toxicology: The Basic Science of Poisons.6th ed. New York: McGraw-Hill Medical Publishing Division. 2001;pp 491-514.

18. Ray SD, Jena N. A hepatotoxic dose of acetaminophen modulates expression of $\mathrm{Bcl}-2$, Blc $-\mathrm{x} \mathrm{L}$, and Bcl - x5 during apoptotic and necrotic cell death of mouse liver cells in vivo. Archives of Toxicology. 2000; 73: $594-606$

19. Boulares AH, Zoltoski A, Yakovlev A, Contreras FJ Smulson ME. Regulation of DNAS1L3 endonuclease ctivity by poly (ADP-ribosylation during etoposide-inched apoptosis: role of PARP-1 cleavage in endonuclease activation. Journal of Biology and Chemistry. 2002, 277: 372-378.

20. Adelman RD, Spangler WL, Beasom F, Ishizaki G, Conzelman GM. Furosemide enhancement of neltimicin nephrotoxicity in dogs. The Journal of Antimicrobial Chemotherapy. 1981; 7(4): 431 - 440.

21. Mayne PD. The kidneys and renal calculi. In: Clinical chemistry in diagnosis and treatment. 6th ed. London: Edward Arnold Publications. 1994; pp 2-24.

Satirapoj B, Supasyndh O, Chaiprasert A, et al. Reationship between serum uric acid levels with chronic kidney disease in a South East Asian population. Nephrology (Carlton). 2010; 15, 253-258.

23. Karadeniz A, Yildirim A, Simsek N, Kalkan Y, and Celebi F. Spirulina platensis protects against gentamicin-induced nephrotoxicity in rats. Phytotherapy Research. 2008; 22, 1506-1510.

24. Miller NJ and Rice-Evans CA. Factors influencing the antioxidant activity determined by the ABTS radical cation assay. Free Radical Research. 1997; 26:195-199. 25. Adeneye AA and Benebo AS. Protective effect of the aqueous leaf and seed extract of Phyllanthus amarus on gentamicin and acetaminophen-induced nephrotoxic rats. Journal of Ethno-pharmacology. 2008; 118:318-323. 26. Oyedapo OO. Biological activity of Phyllanthus armarus extract on Pragrow-Dawley rats. Nigerian Journal of Biochemistry and Molecular Biology. 2001; 26:202-26.

27. Sadique J, Al-Raqobah WA, Bugharith ME, ElGindy AR. The bioactivity of certain medicinal plants on the stabilization of RBC membrane system. Fitoterapia. 1989; 60:525-32.

8. Dorman HJ, Kosar M, Karlos K, Holm Y, Hittune Antioxidant properties and composition of aqueous extracts from Menthaspecies, hybrids, varieties and cultivars. J Agric Food Chem. 2003; 51:4563-9. 